\title{
Categorization of Factors Influencing Community Health Workers from a Socio-Technical Systems Perspective
}

\author{
Lilies Ratshidi ${ }^{1}$ (D), Sara Grobbelaar ${ }^{1,2(\triangle)}(\mathbb{D})$, and Adele Botha ${ }^{1,3}$ (D) \\ ${ }^{1}$ Department of Industrial Engineering, Stellenbosch University, \\ Stellenbosch, South Africa \\ ssgrobbelaar@sun.ac.za \\ 2 DST-NRF Centre of Excellence in Scientometrics and Science, \\ Technology and Innovation Policy (SciSTIP), Stellenbosch University, \\ Stellenbosch, South Africa \\ ${ }^{3}$ CSIR, Pretoria, South Africa
}

\begin{abstract}
In low-and-middle-income countries (LMICs), community health workers (CHWs) are often seen as a connecting bridge between two dynamic and overlapping systems- the community and formal health systems. Although the importance of CHWs is acknowledged, there is minimal aggregated evidence contributing towards understanding their position, technological capabilities, barriers and facilitators of their effectiveness in the South African context. Despite the widespread enthusiasm around the potential that mobile health (mHealth) technology holds in extending healthcare through CHW to underserved communities, an understanding of mHealth's various implications in a developing world context is imperative to appreciate both the community and health systems context. The CHWs within this context need to assume multiple roles as they work and live amongst and in the community. The study argues that by examining their multiple roles as part of the healthcare continuum and from within the community setting, appropriating technological solutions can be conceptualized to facilitate and enhance their impact and visibility. This research article then aims to articulate the key conceptual factors which should be considered when implementing technological solutions for CHWs within the South African context. The aim is operationalized by means of the best-fit framework synthesis method to explore the body of knowledge towards presenting a conceptual understanding through a categorization of Factors Influencing Community Health Workers from a Socio-Technical Systems Perspective.
\end{abstract}

Keywords: Community health worker $\cdot$ Social system $\cdot$ Technical system • Technological solutions $\cdot$ Concepts $\cdot$ Factors

\section{Introduction}

The constitution of South Africa enshrines the provision of healthcare access as a basic human right for all its citizens. However, the South African healthcare system is fraught with challenges, some of the major ones include the inadequacy of human and 
equipment resources [1], difficulties in synergizing and collaborating policies and lack of legislative commitment to improving the public health sector [2]. According to the World Health Organization, the shortage of healthcare workers is a major challenge in a country's ability to overhaul its healthcare system and ultimately, achieve universal healthcare coverage [2]. Given South Africa's limited resources, the need for costeffective strategies is paramount. Recent studies [3,4] identify an adequately competent workforce with multi-faceted roles as having the potential to relieve some of the healthcare system burdens by bridging the healthcare equity gap.

Several authors $[1,2,4]$ have suggested that CHWs as a workforce could be beneficial in the South African context. In addition, it is noted that CHWs are considered a dependable vehicle to provide quality contextual health services both in urban and rural settings within the South African context [5]. The South African government has introduced various initiatives to address the historic disparate healthcare system [1]. One such initiative is the National Development Plan for 2030 that involves building human resources to ensure shared competencies for the health system as part of its plan of action. Part of this plan includes a goal of employing and training between 700000 and 1.3 million CHWs to implement community-based primary healthcare [5]. Regardless of the effort needed to reach this goal quantitively, it can be argued that the potential benefits of CHWs have not to be realized. This can be attributed to a misalignment in national policies and standards to their work practices, and the significant barriers in training CHWs to function at the expected level of competence [2, 6].

With the widespread use of mobile technology in Sub Saharan Africa over the past years, there is an estimated increase to $40 \%$ of mobile internet penetration [7]. There is mounting evidence that suggests the use of mobile health has the potential to enable CHWs to mitigate some of the challenges faced [7]. Consequently, the utilization of technology as a viable solution for CHWs has steadily gained significant popularity. Despite this, various authors' calls for research specifically focused on investigating mHealth implementation for CHWS in low-and-medium-income countries (LMICs). The definition of LMICs adopted was according to the World Bank classification with the study focusing more on the Sub-Saharan region [8]. Winters et al. [9] articulate this as a call for more robust evidence on mobile technology implementation strategies as a means of supporting CHW practices [9]. Granja et al. [10] suggest the successful implementation of technological solutions interventions can be improved through the identification of factors that influences the intervention's outcomes.

From these insights, it can be inferred that an in-depth understanding of the healthcare domain and the processes of technology adoption and use by CHW are a needed step towards achieving the full potential of mHealth. In this regard, various studies have identified factors influencing CHWs. These factors include their perceived performance, motivation and job satisfaction [6, 10]. However, verification of how these factors affect the implementation and evaluation of technological solutions for LMICs has not sufficiently been documented. This study aims to articulate the key conceptual factors which should be considered when implementing technological solutions for CHWs within the South African context. The paper outline is as follows: method, descriptive statistics of the results, discussion and construction of the framework, and the conclusion. 


\section{Method}

\subsection{Methodological Approach}

The study is grounded in social and technical perspectives as it facilitates the duality of the CHWs' work and community role, further adopts the technique of the "best-fit" framework synthesis method in the exploration. The best-fit framework synthesis is defined in [12] as "a means to test, reinforce and build on an existing model, conceived for a potentially different but relevant population". This method involves creating or employing a framework with priori themes and using it to code the data obtained from the relevant studies as a means to produce a rapid and pragmatic form of synthesis [12]. It advises the use of criteria; one for identifying the models and theories to generate a priori framework, and one for populating the scoping review of primary qualitative research studies. In this study, only one set of the literature search and a study selection was considered for the scoping review as the socio-technical system (STS) framework was used as a priori framework. Figure 1 illustrates the methodology approach applied.

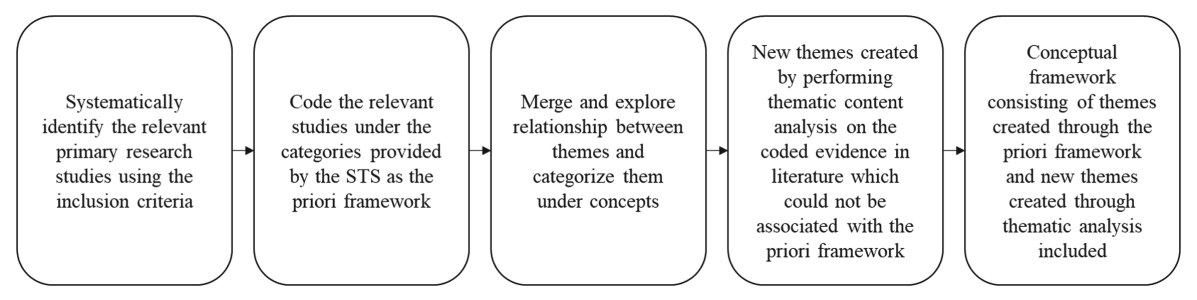

Fig. 1. Methodology approach

\subsection{The Case for STS as a Priori Framework}

Davis et al. [13] describes the STS framework as a system which considers the people involved with distinct social behaviors and skills, working within a physical infrastructure, using a range of technologies and tools to achieve a set of goals and metrics by following sets of processes and practices under a set of cultural assumptions and norms $[12,13]$. STS is defined as an approach to complex work design consisting of technical systems; social systems with an interplay of human agents employing social dependencies that either hold or emerge between them; and finally, organizations that are heterogeneous within unpredictable operational environments, which are autonomous and poorly controllable [14].

The STS theory premises on the combination of social and technical aspects to design a functional work system that can cope with the complexities of the environment within which the system operates in, as well as the dynamics introduced by new technological interventions [15]. Hence, to account for the delicate dynamic relationships within the CHWs' work system, the STS framework was used to diagnose, identify and categorize the literature into the factors and interactions between the social 
and technical elements, and a summary of the study characteristics was transferred to Excel for further synthesis, where they were categorized as either technical or social to generate key inferences regarding the factors which should be considered. The six interrelated elements used are people, infrastructures, goals, technologies, culture, and processes embedded within an external environment [13]. Having overviewed the methodology approach the following section outlines the search strategy employed. In [13], six interrelated elements were presented in the conceptualization of an STS and were used to evaluate the initiatives documented in the literature, from which relevant factors related to each element were identified.

\subsection{Search Strategy and Inclusion Criteria}

A broad literature search was conducted on Scopus, Google, Research Gate, and Google Scholar to identify studies related to CHW initiatives and technology implementation previously conducted in LMICs. The keywords used for the search were: CHWs, framework, technology, healthcare innovation ecosystem, social factors, technical factors, socio-technical systems approach in healthcare, and LMICs. Figure 2 illustrates how the selected inclusion criteria were applied to identify the relevant articles. An iterative process facilitated the addition and removal of studies that were not explicitly addressed by the inclusion criteria. The data extraction process involved recording the full-text articles into publication year, region setting, study type, methodology and key findings after which the six elements were used to identify the relevant factors from the selected literature and categorize them under social or technical perspective [13].

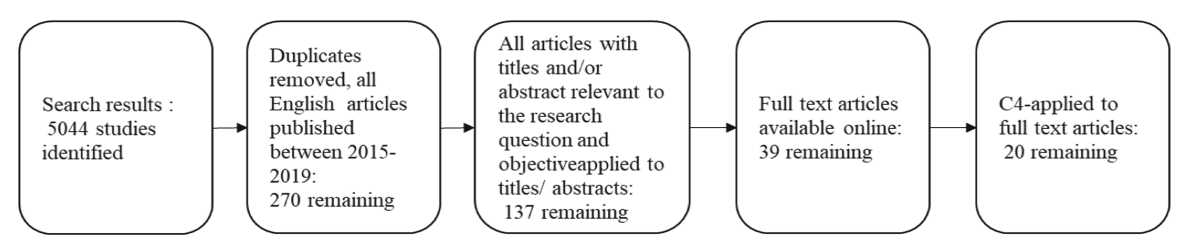

Fig. 2. Flow diagram of the applied inclusion criteria

\section{Descriptive Statistics Findings}

Of the 20 articles included for full-text analysis, most of them used qualitative and mixed methods inquiries involving interviews, focus group discussions with CHWs, healthcare systems stakeholders from government and non-governmental initiatives. The studies were conducted in LMICs including South Africa, Uganda, Ethiopia, Kenya, Mozambique, India, and Zimbabwe. The type of publications resulted in 10 articles on reviews, 6 articles on empirical and 4 on analysis studies. 


\section{Discussion: Synthesis and Categorization of Factors}

The conceptualization of an STS presented in [13] was adapted and applied to the categorization in this study. The following discussion reflects on the analysis and synthesis deduced from the identified literature studies and used to categorize the relevant factors.

\subsection{Social Aspect}

A study by Naimoli et al. [16] posit that the health outcomes achieved through CHWs programs are a function of a robust, high performing health and community sectoral systems. The study further postulates that the programming activities categorized under social, technical and incentives support functions are influenced by a range of contextual factors in both community and health sectoral systems [16]. However, the narrative presented in the previous studies alludes there is unbalanced attention on the impact of the complex and diverse context-specific nature within which CHWs work and live in [17]. As a result, De Neve et al. [17] propose the need for countries to develop coherent and context-specific approaches to ensure optimal performance by CHWs through the consideration of the broader context, including demographic, socioeconomic, political, legislative, ecological, sociocultural, and technological factors contributing towards facilitating or inhibiting the success of many CHWs initiatives [18, 19]. Some of the solutions to achieve what is postulated in the studies include coordinating the health system and community system to prioritize factors that inhibit or facilitate the understanding of CHWs programs' compatibility with community structures, cultural values, and perception, socio-economic context and support system [20].

In addition, integrating and adopting interventions supported by technological solutions, and the sustainability of these interventions should be considered when exploring efforts until the desired health outcomes are achieved to gain a better understanding of CHWs programs and their roles in LMICs [17]. Previous research that was focused on CHWs and their performance placed emphasis on developing frameworks that provide a broad context of the CHW's position in a larger environment by describing the interrelations of intrapersonal, family, community, and organizational settings as health professionals $[6,10]$. This perspective to a larger extent provides a limited understanding of the impact of the ecological environment on CHWs [20]. Most programs have not been able to effectively address the gap between research evidence and the routine practicality of CHWs as health professions, hence the poor integration of CHWs within the healthcare system and an even poorer understanding of their roles within their communities. Subsequently, the implication of this postulates the need for a comprehensive approach to plan and design programs that can be integrated with the formal healthcare system's approach to healthcare service delivery through CHWs roles and organizations [17].

Moreover, the CHW system requires an interface with the formal healthcare and the community systems involving the political structures, civic groups, faith-based organizations. Schneider and Lehmann [21] argue that integrating CHWs into the primary 
healthcare systems while embedding and supporting them through the community is vital to realize their potential. Contrarily, most studies emphasize the need for CHWs to be integrated within the formal healthcare system whilst placing minimal emphasis on understanding how they are embedded within the community system [21]. Moreover, minimal work has been done locally in terms of implementing universal guidelines to guide the integration strategies required to resolve the above-stated implications. Previous research has proved that the effectiveness of $\mathrm{CHWs}$ holds the potential to increase access to equitable health in LMICs [20]. As a result, CHWs' understanding of the socio-cultural norms of their communities, their unique intermediary position between communities and the health system places them in a central setting in delivering key health interventions. On an individual level, their effectiveness is influenced by contextual factors, such as socio-cultural factors, gender, traditions and norms, training and supervision, health policies combined with intervention-related factors [22].

Furthermore, it is postulated in [16] that support for CHWs has to be strategic, collaborative and well-coordinated to enhance CHWs performance between the two overlapping dynamic systems they are expected to function within. Among the key challenges presented in the study, the definition and optimization of the impact of the CHW' roles are highlighted as an influence on $\mathrm{CHW}$ performance. Also, [3] argues that government and non-governmental institutions are continuously adding functions and tasks to CHWs, which buttresses the need to inform the type of tasks and position they hold within the healthcare system through competency-based or educational qualification rather than on functionalities. In most LMICs such as South Africa, the roles and responsibilities of CHWs with regards to technical and social capital is limited and yet to be understood [11]. Khalala et al. [23] state that understanding the nature of the work CHWs do, enables researchers to explore the relevant technologies that can be exploited to facilitate and support their daily work. This understanding potentially provides information with regards to the choice of technology and how it can be implemented to support the roles of CHWs. Regardless of the evidence on the social factors influencing CHWs, the exact mechanisms on how to assess the interdependencies of social and technical dynamics' influence on CHWs outcomes remains understudied.

\subsection{Technical Aspect}

Technologies are not neutral or passive objects but rather shape the environment and provoke social dynamics as a result of their existence and necessity for human survival. Previous studies reveal that technologies have the potential to influence social, cultural and economic contexts and improve healthcare quality for communities when employed in the healthcare domain [24]. Expectedly, the use of mobile technology in South Africa in the healthcare system has also increased, particularly among CHWs [25]. Despite CHWs having limited formal education and training, with poorly defined roles in using technologies within their line of work, it has been emphasized in [4] that most research has focused on the usability and reliability of technologies with minimal emphasis on the users and the important aspect that they are social beings who interact 
with their immediate and remote communities. Iluyemi et al. [26] state that to conceptualize technological solutions and their policy interventions, it is important to start by gaining an understanding of the context of use and needs for the technology from the CHWs' perspective. In addition, the challenges of technology's usability and supportive structures. Existing literature have focused on the reliability, functionality and infrastructure of the technology while paying minimal attention to the end-users perception on the usability and intentions to use the technology, as a result most of the research focus on the technical characteristics whilst neglecting the impact of the social characteristics of both the individual and community setting where the technology is utilized [27].

Nonetheless, this implies there is a need to conceptualize the appropriate technologies which can both fit the task they are used to as well as have the capabilities to perform the task. Moreover, technology and policy interventions are deemed as necessary developments to enhance technological effectiveness and efficiency, and to ensure sustainability and scalability through the initiatives whilst amplifying their impact [28]. Thus, the technology acceptance dimensions are considered in describing the understanding of the technological capabilities of CHWs, their ability to access and utilize the appropriate technologies applicable to their social backgrounds. There is undeniable evidence from previous studies about the importance of considering the sociotechnical determinants in developing implementation and evaluation conceptual models for technological solutions. Determinants including technological appropriateness and socio-cultural sensitivity, political infrastructures, the technology endusers' attributes and variables of ecological settings [29]. In a formative study about the adoption and usage of mHealth by CHWs in India, Kaphle et al. [20] hypothesized that individual characteristics of end-users such as education, experience of care, and demographics hold the potential to influence the uptake of technological adoptions and quality of care. In addition, Kim et al. [30] reinforces that the attitude of health professions to use technology influences the behavioral intention to use it.

As a result, the relationship between technology and CHWs' performance is associated with their readiness to align their behavioral intention to use the technology. Important to note, technological solutions and processes are not autonomous vehicles, but rather are embedded within systems in a social world where they are used to perform activities which have consequences and influences changes on human behavior, social constructs, and cultural meanings. Thus, the effect of this is that not only does the interaction between the technical and social systems exhibit complexity and unpredictable behavior; it inevitably increases the complexities of dynamic and autonomous relationships, which can be detrimental to the system in place. From the above sections, the following is presented in summary to illustrate the factors identified in the discussion using the STS hexagon for illustration as provided in [13]. Figure 3 illustrates the social and technical perspectives identified from previous literature according to [13]. 


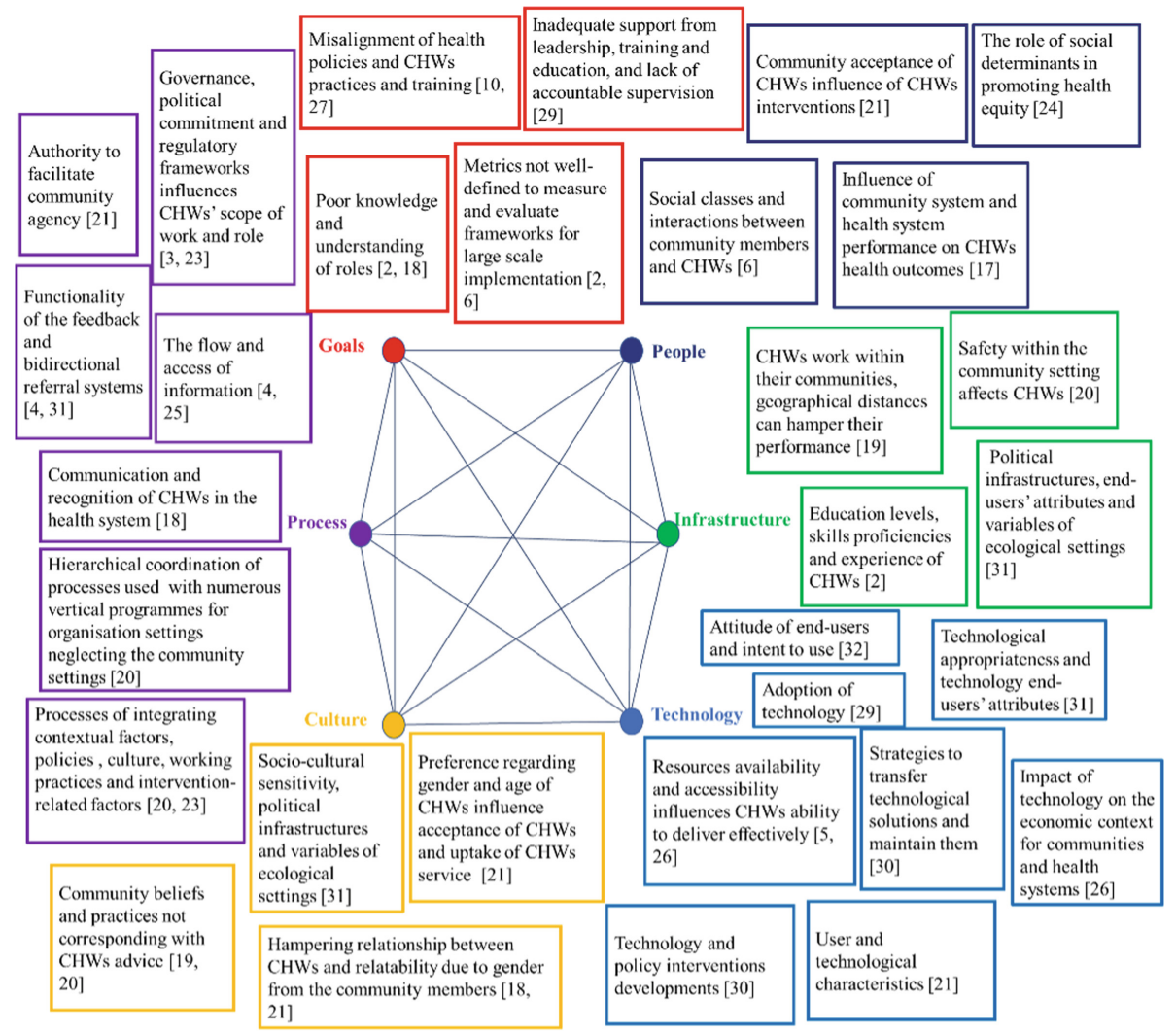

Fig. 3. Identified factors influencing CHWs using STS perspective

\subsection{Integrating Factors into Concepts}

From the discussion above, the factors (see Fig. 3) were categorized into concepts according to their similarities to allow for a reasonable number of concepts to be considered. The approach followed in this study desired that the design activities involve gaining understanding and specification of the context in which the system will be used by referring to the social factors, cultural factors, working practices factors, and the structure of the organization [31]. Thus, the terminologies for the concepts were adapted from this standpoint and were cautiously selected by the author through interpretation about the relevant literature as a means to preserve the relevance of the factors. The concepts were grouped under technical and social context depending on the interpretation of the author, from there, they were categorized under the two social dimensions: health and community systems context depending on whether the influence of the factor on the CHWs was related and reflective of the context in question. This study considers the following fundamental areas under the social system: the individual's needs, humans' social behaviors in work systems, internal and external environmental factors and support systems of the work system under investigation [15]. 
Whilst the technical system focuses on the processes, tasks, infrastructures, and technologies required and used in the work system to achieve the set goals [14]. Additionally, the technical dimensions included technology readiness which was concerned with categorizing the factors related to the technological solutions characteristics and compatibility to be used. The second dimension was technology acceptance and utilization which was concerned with factors concerning the CHWs perspectives and readiness to use and accept the technological solution within the environment they work. Finally, the compatibility/fit element was considered as a means to provide the measures required to ensure a balanced joint optimization of both the technical and social aspects [31]. Figure 4 illustrates the concepts classed under social and technical context.

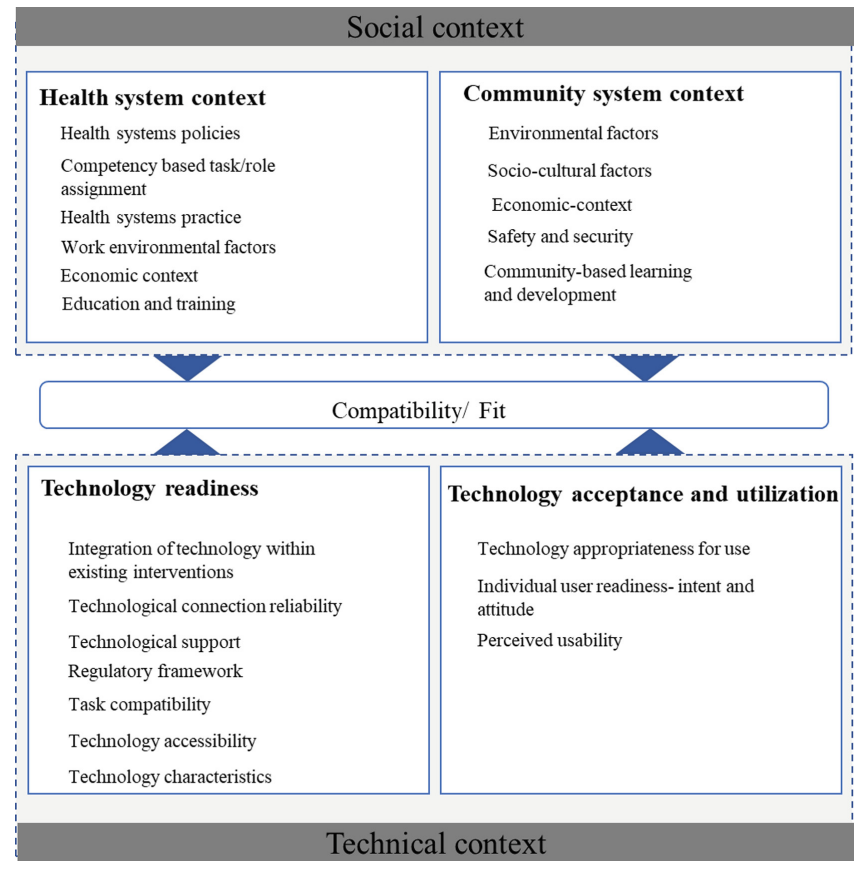

Fig. 4. Overview of the factors categorized as concepts

\section{Conclusion}

This research article aimed to articulate the key conceptual factors which should be considered when implementing technological solutions for CHWs within the South African context. The STS framework was used for the categorization of the factors which were integrated into concepts. For future work, each of these concepts will be evaluated and the categorization refined to present a broader conceptual framework. This study forms part of a dissertation project and the findings in this study form part of the first high-level conceptual framework which will be used in conducting scoping literature review to identify additional components for the secondary-level conceptual 
framework. In addition, future plans involve exploring the task-technology fit model to identify the relevant technological solutions fit for peri-urban and rural contexts in South Africa.

\section{References}

1. Maphumulo, W.T., Bhengu, B.R.: Challenges of quality improvement in the healthcare of South Africa post-apartheid: a critical review. Curationis 42(1), 1-9 (2019)

2. White, M.S., Govender, P., Lister, H.E.: Community health workers lensed through a South African backdrop of two peri-urban communities in KwaZulu-Natal. African J. Disabil. 6, 18 (2017). https://doi.org/10.4102/ajod.v6i0.294

3. Olaniran, A., Smith, H., Unkels, R., Bar-Zeev, S., van den Broek, N.: Who is a community health worker? - A systematic review of definitions. Glob. Health Action 10(1), 1272223 (2017). https://doi.org/10.1080/16549716.2017.1272223

4. Early, J., Gonzalez, C., Gordon-Dseagu, V., Robles-Calderon, L.: Use of mobile health (mHealth) technologies and interventions among community health workers globally: a scoping review. Health Promot. Pract. 20, 1-13 (2019). https://doi.org/10.1177/152483 9919855391

5. van Heerden, A., et al.: Perceived mHealth barriers and benefits for home-based HIV testing and counseling and other care: qualitative findings from health officials, community health workers, and persons living with HIV in South Africa. Soc. Sci. Med. 183, 97-105 (2017). https://doi.org/10.1016/j.socscimed.2017.04.046

6. Zalani, G.S., et al.: Affecting factors on the performance of community health workers in Iran's rural areas: a review article. Iran J. Public Health 45(11), 1399-1410 (2016)

7. Aranda-Jan, C.B., Mohutsiwa-Dibe, N., Loukanova, S.: Systematic review on what works, what does not work and why of implementation of mobile health (mHealth) projects in Africa. BMC Public Health 14(188), 1-15 (2014)

8. World Bank Country and Lending Groups - World Bank Data Help Desk. https:// datahelpdesk.worldbank.org/knowledgebase/articles/906519. Accessed 06 Jan 2020

9. Winters, N., et al.: Using mobile technologies to support the training of community health workers in low-income and middle-income countries: mapping the evidence. BMJ Glob. Heal. 4, 1-10 (2019). https://doi.org/10.1136/bmjgh-2019-001421

10. Granja, C., Janssen, W., Johansen, M.A.: Factors determining the success and failure of ehealth interventions: systematic review of the literature. J. Med. Internet Res. 20(5), 1-21 (2018). https://doi.org/10.2196/10235

11. Buehler, B., Ruggiero, R., Mehta, K.: Empowering community health workers with technology solutions. IEEE Technol. Soc. Mag. 32(1), 44-52 (2013). https://doi.org/10. 1109/mts.2013.2241831

12. Carroll, C., Booth, A., Leaviss, J., Rick, J.: "Best fit" framework synthesis: refining the method. BMC Med. Res. Methodol. 13(1), 13-37 (2013). https://doi.org/10.1186/14712288-13-37

13. Davis, M.C., Challenger, R., Jayewardene, D.N.W.W., Clegg, C.W.: Advancing sociotechnical systems thinking: a call for bravery. Appl. Ergon. 45(2), 171-180 (2014). https:// doi.org/10.1016/j.apergo.2013.02.009

14. Dalpiaz, F., Giorgini, P., Mylopoulos, J.: Adaptive socio-technical systems: a requirementsbased approach, 18, 1-24 (2013). https://doi.org/10.1007/s00766-011-0132-1 
15. Mwendwa, P.: What encourages community health workers to use mobile technologies for health interventions? Emerging lessons from rural Rwanda. Dev. Policy Rev. 36, 111-129 (2018). https://doi.org/10.1111/dpr.12275

16. Naimoli, J.F., Perry, H.B., Townsend, J.W., Frymus, D.E., McCaffery, J.A.: Strategic partnering to improve community health worker programming and performance: features of a community-health system integrated approach. Hum. Resour. Health 13(46), 1-13 (2015). https://doi.org/10.1186/s12960-015-0041-3

17. De Neve, J.W., et al.: Harmonizing community-based health worker programs for HIV: a narrative review and analytic framework. Hum. Resour. Health 15(1), 1-10 (2017). https:// doi.org/10.1186/s12960-017-0219-y

18. Kambarami, R.A., Mbuya, N.N., Pelletier, D., Fundira, D., Tavengwa, N.V., Stoltzfus, R.J.: Factors associated with community health worker performance differ by task in a multitasked setting in rural Zimbabwe. Glob. Health Sci. Pract. 4(2), 238-250 (2016)

19. Kok, M.C., et al.: Which intervention design factors influence performance of community health workers in low- and middle-income countries? A systematic review. Health Policy Plan. 30(9), 1207-1227 (2015). https://doi.org/10.1093/heapol/czu126

20. Kaphle, S., Chaturvedi, S., Chaudhuri, I., Krishnan, R., Lesh, N.: Adoption and usage of mHealth technology on quality and experience of care provided by frontline workers: observations from rural India. JMIR mHealth uHealth 3(2), e61 (2015). https://doi.org/10. 2196/mhealth.4047

21. Schneider, H., Lehmann, U.: From community health workers to community health systems: time to widen the horizon? Health Syst. Reform 2(2), 112-118 (2016). https://doi.org/10. 1080/23288604.2016.1166307

22. Saprii, L., Richards, E., Kokho, P., Theobald, S.: Community health workers in rural India: analysing the opportunities and challenges Accredited Social Health Activists (ASHAs) face in realising their multiple roles. Hum. Resour. Health 13(95), 1-13 (2015). https://doi.org/ 10.1186/s12960-015-0094-3

23. Khalala, G., Makitla, I., Botha, A., Alberts, R.: The roles and needs of community health workers in developing countries: an exploratory case study in South Africa. In: IEEE International Conference on Adaptation Science Technology ICAST, pp. 1-5 (2013). https:// doi.org/10.1109/icastech.2013.6707498

24. Grover, P., Kar, A.K., Davies, G.: "Technology enabled health" - Insights from twitter analytics with a socio-technical perspective. Int. J. Inf. Manag. 43, 85-97 (2018). https://doi. org/10.1016/j.ijinfomgt.2018.07.003

25. Lindberg, M., Rosborg, S., Ramukumba, M.M., Hägglund, M.: Adapting mHealth to workflow - a case study in South Africa. Stud. Health Technol. Inform. 265, 48-53 (2019). https://doi.org/10.3233/SHTI190136

26. Iluyemi, A., Briggs, J., Adams, C.: Mobile information system, health work and community health workers in less developed countries. In: Proceedings of Workshops on Enabling Technologies: Infrastructure for Collaborative Enterprises, WETICE, pp. 208-209 (2007). https://doi.org/10.1109/wetice.2007.4407155

27. Winters, N., Langer, L., Geniets, A.: Scoping review assessing the evidence used to support the adoption of mobile health (mHealth) technologies for the education and training of community health workers (CHWs) in low-income and middle-income countries. BMJ Open 8(7), 1-10 (2018). https://doi.org/10.1136/bmjopen-2017-019827

28. Gagnon, M.P., Ngangue, P., Payne-Gagnon, J., Desmartis, M.: M-Health adoption by healthcare professionals: a systematic review. J. Am. Med. Inform. Assoc. 23(1), 212-220 (2016). https://doi.org/10.1093/jamia/ocv052 
29. Aamir, J., Ali, S.M., Kamel Boulos, M.N., Anjum, N., Ishaq, M.: Enablers and inhibitors: a review of the situation regarding mHealth adoption in low- and middle-income countries. Health Policy Technol. 7, 88-97 (2018). https://doi.org/10.1016/j.hlpt.2017.11.005

30. Kim, S., Lee, K., Hwang, H., Yoo, S.: Analysis of the factors influencing healthcare professionals' adoption of mobile electronic medical record (EMR) using the unified theory of acceptance and use of technology (UTAUT) in a tertiary hospital. BMC Med. Inform. Decis. Mak. 16, 1-12 (2016). https://doi.org/10.1186/s12911-016-0249-8

31. Baxter, G., Sommerville, I.: Socio-technical systems: from design methods to systems engineering. Interact. Comput. 23(1), 4-17 (2011). https://doi.org/10.1016/j.intcom.2010.07. 003 